ISSN 0103-5150

Fisioter. Mov., Curitiba, v. 24, n. 2, p. 307-314, abr./jun. 2011 Licenciado sob uma Licença Creative Commons

\title{
Prevalência de sintomas osteomusculares em professores de escolas públicas e privadas do ensino fundamental
}

\author{
Prevalence of musculoskeletal symptoms in the faculty of public \\ schools and the private school
}

\author{
Jerônimo Costa Branco ${ }^{[a]}$, Felipe Guido e Silva ${ }^{[b]}$, Karen Jansen ${ }^{[c]}$, Patrícia Haertel Giusti ${ }^{[d]}$ \\ [a] Doutorando em Saúde e Comportamento na Universidade Católica de Pelotas (UCPEL), Pelotas, RS - Brasil, e-mail: \\ jeronimobranco@hotmail.com \\ [b] Graduado em Fisioterapia na Universidade Católica de Pelotas (UCPEL), Pelotas, RS - Brasil, e-mail: felipeguido@brturbo.com.br \\ [c] Mestre em Saúde e Comportamento na Universidade Católica de Pelotas (UCPEL), Pelotas, RS - Brasil, e-mail: \\ karenjansen315@hotmail.com \\ [d] Mestre em Saúde e Comportamento na Universidade Católica de Pelotas (UCPEL), Pelotas, RS - Brasil, e-mail: \\ phgiustia@gmail.com
}

\section{Resumo}

Objetivos: Diante da preocupação com a saúde e as condições de trabalho dos professores, este artigo objetivou verificar a prevalência de sintomas osteomusculares em professores do ensino fundamental de escolas públicas e privadas. Métodos: A amostra foi composta por 320 professores de seis escolas. Para a pesquisa, fez-se uso de um questionário sociodemográfico, de um check-list para avaliação ergonômica, e do questionário nórdico para avaliar os sintomas osteomusculares. Para análise dos dados utilizou-se o programa SPSS ${ }^{\circledR}$ 10.0. Resultados: Dos participantes, 89,7\% referiram algum sintoma nos últimos 12 meses. As áreas mais acometidas por estes sintomas foram: coluna dorsal 54,1\% (173), pescoço 50,9\% (163) coluna lombar 49,1\% (157). Os sintomas impossibilitaram 36,6\% dos professores de exercer suas atividades normais. Observou-se também que a condição ergonômica do local de trabalho é considerada ruim pelos professores. Conclusão: Concluímos que a prevalência de sintomas osteomusculares nos professores é alta e as condições ergonômicas são consideradas inapropriadas, podendo interferir diretamente em sua saúde.

Palavras-chave: Docentes. Sistema musculosquelético. Ergonomia. Saúde do trabalhador. Fisioterapia. 


\section{Abstract}

Purpose: Due to the concern with the health and working conditions of teachers in the urban area of Pelotas, $R S$, the purpose of this study is to verify the prevalence of ostheomuscular symptoms in public and private elementary school teachers. The sample was composed by 320 teachers in six schools. Method: For accomplishment of the research, a social-demographic questionnaire, a check-list for ergonomic evaluation, and the Nordic questionnaire were applied in order to evaluate the ostheomuscular symptoms. For analysis of the data the SPSS 10.0 program was used. Results: 89,7\% of the participants referred some symptom in the last 12 months. The most affected areas were: spine 54,1\% (173), neck 50,9\% (163) lumbar column 49,1\% (157). The symptoms disabled $36,6 \%$ of the teachers hindering them from working properly. It was also observed that the ergonomic condition of the work place was considered unsatisfactory by the teachers. Conclusion: The study concluded that the prevalence of ostheomuscular symptoms among teachers is high, and that the ergonomic conditions are considered inappropriate which could interfere directly in their health.

Keywords: Teachers. Musculoskeletal system. Ergonomics. Occupational health. Physio therapy.

\section{Introdução}

Um dos maiores desafios do século atual, em que as pessoas estão em busca de estabilidade e reconhecimento profissional, está em aprender a conviver neste mundo globalizado, e a educação emerge um grande trunfo, por possibilitar o desenvolvimento contínuo de pessoas e da sociedade. No entanto, a falta de recursos materiais, condições de trabalho inadequadas e o acúmulo de exigências levam à sobrecarga dos professores. 0 encontro da prática é distante dos ideais pedagógicos assimilados durante o período de formação, uma divergência que incide diretamente sobre a ação docente, gerando tensões sobre a saúde $(1,2)$.

A docência é uma das mais antigas ocupações. 0 docente, anteriormente visto como uma figura essencial para a sociedade, hoje é um trabalhador que luta pela valorização e o reconhecimento social (3). No Brasil, o dado mais recente, publicado em 2003 pelo Instituto Nacional de Estudos e Pesquisas Educacionais Anísio Teixeira, aponta que o número de funções docentes é de 2.497,918 (4).

A despeito da carga horária de trabalho com média de 40 horas semanais, muitos professores levam para casa atividades que deveriam ser executadas dentro das horas já mencionadas - o que ocasiona efeitos nocivos sobre a sua condição de saúde, uma vez que isso pode interferir em seu lazer (5). Este trabalho excessivo e pouco remunerado pode favorecer o aparecimento de sintomas osteomusculares (6).

Estudos comprovam alta prevalência desses sintomas em trabalhadores, e os professores estão entre os profissionais mais acometidos, alcançando 93,0\% (7). Muitos desses acarretam o afastamento do trabalho, por quadros álgicos intensos ou pelo desenvolvimento de doenças ocupacionais. De acordo com o Centro de Estudos da Saúde do Trabalhador (CESAT) de Salvador, BA, 66\% dos professores atendidos entre os anos de 1995 a 2001 foram diagnosticados com doença ocupacional (8).

Muitas organizações, incluindo o ambiente escolar, não oferecem condições ergonômicas adequadas, que estejam em conformidade com a legislação trabalhista e, em especial, com o que dispõe a norma regulamentadora 17 (NR 17), a qual preconiza estabelecer parâmetros adaptativos que garantam condições adequadas de trabalho (9). Diante disto, os sintomas osteomusculares têm sido uma preocupação dos pesquisadores, pois se trata de uma questão de saúde e trabalho, em virtude dos custos e o impacto na qualidade de vida dos trabalhadores $(7,9,10)$.

Este estudo teve por objetivo verificar a prevalência de sintomas osteomusculares em professores do ensino fundamental da rede publica e privada da zona urbana de Pelotas, RS.

\section{Método}

Foi realizado um estudo transversal com professores do ensino fundamental de escolas públicas e privadas na zona urbana de Pelotas no ano de 2009.

Inicialmente foi realizado um contato prévio com o Sindicato dos Professores do Rio Grande do Sul (SINPRO-RS) e a Secretaria Municipal de Educação 
(SME) para verificar a viabilidade da realização do estudo e conceder-nos a autorização.

A amostra foi calculada utilizando o Epi Info ${ }^{\circledR}$ 6.0, segundo dados do SINPRO-RS e SME, havendo um total de 3.413 professores de ensino fundamental na cidade de Pelotas. Seria necessário estudar 257 professores, considerando um intervalo de confiança de 95\%, prevalência media de sintomas osteomusculares de $92 \%(7,11)$ e margem de erro de 3 pontos percentuais. Adicionando-se 10\% para perdas e recusas, totalizou-se uma amostra mínima de 283 professores.

Fizeram parte da amostra 320 professores, sendo entrevistados todos os professores de seis escolas - as três maiores escolas privadas e as três maiores municipais, levando em consideração o número de alunos matriculados no ensino fundamental no ano de 2009. Os professores deveriam estar trabalhando diretamente com os alunos em sala de aula. Foram excluídos do estudo os docentes que estivessem afastados, com licença ou que não compareceram à escola durante o período de coleta de dados, além dos estagiários. Essas entrevistas foram realizadas nas escolas selecionadas no período do intervalo entre as aulas.

0 perfil dos professores foi coletado a partir de um instrumento formulado pelos autores deste projeto. As informações referentes aos sintomas osteomusculares foram coletados por meio do Questionário Nórdico de Sintomas Osteomusculares (QNSO) (12). Este incide em escolhas múltiplas ou binárias quanto à ocorrência de sintomas nas diversas regiões anatômicas do corpo nos doze últimos meses e nos últimos sete dias, bem como o afastamento de suas atividades normais (12).

A avaliação do ambiente físico de trabalho foi realizada por um check-list proposto por Couto (1995), adaptado pelos autores do projeto, para os entrevistados responderem, em vez de serem avaliados pelos pesquisadores (13).

Os questionários, depois de revisados e codificados, foram digitados utilizando o programa Epi-Info ${ }^{\circledR}$ versão 6.0, com realização de dupla digitação, para checagem automática da consistência dos dados. A análise dos dados foi no programa SPSS ${ }^{\circledR}$ versão 10.0. Para tal, foi realizada frequência simples das variáveis independentes e desfecho, além da associação entre estes por meio do teste qui-quadrado, que foi considerado estatisticamente significativo quando $\mathrm{p} \geq 0,05$.
Nesta pesquisa foram respeitados todos os princípios éticos estabelecidos pelo Conselho Nacional de Saúde na Resolução n. 196, de 10 de outubro de 1996. 0 estudo foi aprovado pelo protocolo 2008/ 102 do Comitê de Ética da Universidade Católica de Pelotas. Os professores receberam informações sobre os objetivos da pesquisa e assinaram o Termo de Consentimento Livre e Esclarecido.

\section{Resultados}

Dos 355 professores das seis escolas selecionadas, 90,1\% (320) participaram do estudo, dos quais 35 não participantes, 23 foram perdas e 12 recusas. Do total pesquisado, 25\% (80) trabalhavam em escolas particulares e 75\% (240) em públicas; nas públicas, a prevalência dos sintomas foi de $91,7 \%$ e nas privadas, de $83,8 \%(p=0,129)$. A prevalência geral de sintomas osteomusculares referido pelos docentes nos últimos doze meses foi de $89,7 \%$ (287) e de 68,4\% (219) nos últimos sete dias.

A prevalência de sintomas osteomusculares em professores do sexo masculino foi de $91,3 \%$ (42) e no sexo feminino de 89,4\% (245) ( $p=0,469)$. Com relação ao tempo de serviço e à presença de sintomas osteomusculares, foi observado que 34,8\% trabalhavam de 1 a 8 anos, 33,4\% de 9 a 19 anos e $31,7 \%$ de 20 a 41 anos ( $\mathrm{p}=0,156$ ).

Dos professores que apresentavam algum tipo de sintoma, $35,5 \%$ tinham idade entre 21 e 35 anos, $33,1 \%$ tinham de 36 a 44 anos e 31,4\% tinham de 45 a 69 anos $(p=0,498)$. Apesar da idade não apresentar associação significativa com a presença de sintomas, os professores de mais idade apresentaram maior número de sintomas osteomusculares em membros inferiores: $60,2 \%(p=0,01)$.

Na relação dos sintomas osteomusculares com a carga horária, os professores que trabalhavam de 1 a 20 horas semanais apresentaram uma prevalência de $20,6 \%$, aqueles que realizavam de 20 a 40 horas apresentaram $52,6 \%$, enquanto os trabalhadores de 41 a 60 horas apresentaram $26,8 \%$ de sintomas osteomusculares. A presença de sintomas, apesar de apresentar diferentes proporções entre os grupos, não apresentou associação estatisticamente significativa $(\mathrm{p}=0,33)$.

A fim de verificar a prevalência por segmento mais afetado, o corpo humano foi agrupado em três regiões (coluna vertebral, membros inferiores e membros 
superiores). Do total de professores que relataram a presença de sintomas osteomusculares em alguma parte do corpo, 79,7\% apresentaram na coluna vertebral, sendo esta o maior alvo de queixas, seguida pela região dos membros superiores $(67,4 \%)$ e da região dos membros inferiores (45,9\%). A coluna dorsal foi o local mais acometido, com 54,1\% (173), seguido pelo pescoço, com 50,9\% (163), e pela lombar, com 49,1\% (157) (Tabela 1 - anexo).

No que concerne à capacidade funcional, $36,6 \%$ (105) não conseguiram realizar suas atividades normais nos últimos doze meses graças à presença dos sintomas $(\mathrm{p}=0,00)$ (Tabela 1$)$.

Dos 320 professores, $74,1 \%$ (237) realizavam atividade extraclasse, dos quais $91,56 \%$ (215) referiram sintoma osteomuscular nos últimos doze meses.

Diante da avaliação ergonômica, constatou-se que quanto pior as condições de trabalho, mais os sintomas osteomusculares são sentidos pelos professores $(p=0,003)$. Nas escolas privadas 32,5\% (26) e nas públicas 45,8\% (110) consideram as condições de trabalho ruins (Tabela 2 - anexo).

Dos professores que relataram queixas de sintomas osteomuscular nos últimos doze meses e nos últimos sete dias, respectivamente $74,9 \%(\mathrm{p}=0,00) \mathrm{e}$ $79,5 \%(p=0,00)$ acreditam que a sintomatologia está relacionada ao trabalho que realizam como docente.

No que diz respeito à prevalência de sintomas osteomusculares em relação à disciplina ministrada, os professores de Educação Física foram os que menos apresentaram: 18,2\% (26) deles não relataram sintomas nos últimos doze meses. Entretanto, os que mais relataram os sintomas foram professores de Educação Artística, com 100\% (17) de queixas. Este dado, embora não apresente associação estatisticamente significativa, demonstra a grande prevalência de sintomas nesta classe de professores (Tabela 3 - anexo).

\section{Discussão}

No presente estudo procurou-se ampliar os conhecimentos sobre as queixas músculo-esqueléticas em professores do ensino fundamental do município de Pelotas, RS.

Os resultados demonstram elevada ocorrência de sintomas osteomusculares em professores do ensino fundamental, sendo uma prevalência geral de $89,7 \%$ nos últimos 12 meses. Dados semelhantes a este foram observados em um estudo realizado no interior de São Paulo com professores da rede pública do ensino fundamental, em que as queixas foram 90,4\% (11). Também em estudos com trabalhadores de diferentes áreas encontrou-se uma média de $79,58 \%$ de queixa de sintomas osteomusculares (11, 14-17).

A alta prevalência encontrada pode estar associada a diversos fatores do dia a dia de trabalho desta classe de professores. Podemos destacar o fato de trabalhar muitas horas com o membro superior suspenso associado à rotação de tronco com o pescoço levemente inclinado propiciando à musculatura cervical, escapular e tóraco-lombar desenvolver sintomas dolorosos $(18,19)$. Além disso, a pouca movimentação no ambiente de trabalho possibilita a execução do trabalho na posição estática, que embora possa não ser tão intensa, se prolongada e associada à inércia muscular pode produzir fadiga $(19,20)$.

Na presente pesquisa encontrou-se que os professores de colégios públicos apresentam mais sintomas que os de escolas privadas, embora não se verifique associação estatística sugerindo que, independentemente do tipo de instituição em que lecione suas aulas, a prevalência dos sintomas seja alta e semelhante. Embora na maioria das escolas privadas os recursos para a prática laboral, como quadros branco, datashow e armários sejam melhores (8), em nossa amostra estes recursos não possibilitaram a diminuição na prevalência de sintomas.

Hoje a docência é uma atividade exercida na maioria das vezes por mulheres. Sabe-se que o trabalho feminino sofreu transformações, que seu papel deixou de ser somente o cuidar da casa e dos filhos, ocorrendo a emancipação da mulher $(21,22)$.

Neste estudo, embora o número de homens participantes (46) seja inferior ao de mulheres (274), foi encontrada maior prevalência de sintomas osteomusculares no sexo masculino (91,3\%). Em contrapartida, os estudos apresentam que a maior prevalência de sintomas osteomusculares é no sexo feminino $(7,14,16)$.

Ao analisarmos o tempo de serviço e a idade associado aos sintomas, verificou-se que quanto menor o tempo de serviço e quanto menor a idade, maior foi a prevalência de sintomas, dados também encontrados em um estudo realizado com professores do interior de São Paulo (23). Isto se deve provavelmente à não adaptação à sua nova fase ocupacional, sendo 
inversamente ao esperado entre o tempo de exposição e a presença do sintoma.

Verificou-se em nosso estudo que quanto mais avançada a idade, maior é a presença de sintomas ostemusculares nos membros inferiores, com uma prevalência de $60 \%$ de queixas. Durante o envelhecimento, a elasticidade dos tendões, ligamentos e cápsulas articulares diminuem por causa de deficiências do colágeno, acarretando restrição da mobilidade, amplitude de movimentos e flexibilidade das articulações. A sobrecarga das articulações, em particular aquelas que suportam peso e fazem movimentos em excesso, provoca um processo degenerativo de desgaste da cartilagem, ocasionando dor (24).

Os resultados obtidos em nosso estudo corroboram com os encontrados em um estudo realizado com professores de Ponta Grossa no que diz respeito à carga horária semanal de trabalho. Os professores que trabalham de $21 \mathrm{~h}$ a $40 \mathrm{~h}$ são os que apresentam mais queixas de sintomas osteomusculares (25). Acredita-se que os professores que trabalham até $20 \mathrm{~h}$ possuem mais tempo para atividades de lazer, os de mais de $41 \mathrm{~h}$ não possuem tempo de realizar outras atividades, e os de $21 \mathrm{~h}$ a $40 \mathrm{~h}$, além de trabalhar, podem realizar atividades extras conjuntamente, implicando sobrecarga física.

Em relação ao segmento mais acometido pelos participantes desta pesquisa destacamos o da coluna vertebral - a coluna dorsal é aquela a que se relacionou o maior número de queixas. Resultados semelhantes são encontrados em pesquisas realizadas com professores, enfermeiros e metalúrgicos, porém o segmento da coluna vertebral mais referido foi a região lombar $(11,15,16)$. Uma alta porcentagem dos professores associa suas queixas posturais a ter que manter uma posição inadequada e incômoda do corpo durante o trabalho, além de necessitar da realização do esforço físico na sua prática profissional $(18,26,27)$.

0 grande número de docentes que relataram à incapacidade de realizar algum tipo de suas atividades normais pode estar associado a inúmeros fatores ocorridos dentro e fora da escola. A alta prevalência de sintomas osteomusculares, as condições ergonômicas insatisfatórias e o grande número de profissionais que desempenham alguma atividade além da docência podem contribuir para esta incapacidade.

A carga de trabalho atribuída aos professores compreende o tempo de preparação das aulas, das tarefas exercidas dentro dos colégios, ligadas à organização social escolar, além da fastidiosa correção de provas, mas diante da realidade dos salários defasados, muitos deles estão recorrendo a trabalhar em dois turnos na escola e/ou fazer atividades diferentes de sua profissão para conseguir melhorar a renda mensal (28-30). Diante desta realidade foi verificado em nosso estudo que a prevalência de sintomas em professores que realizam algum tipo de atividade extraclasse é alta: dos 237 professores que tinham outra atividade, 215 (91,56\%) referiram a presença de algum sintoma.

No que diz respeito à matéria lecionada, os professores de Educação Física são os que relataram menos dor músculo-esquelética. Acredita-se que isto está relacionado à melhor percepção de seu corpo e fato de esses profissionais possuírem o conhecimento da prática e da importância da atividade física. Os que desempenham atividade de professor ainda desfrutam de um ambiente de trabalho propício à prática de exercícios, alongamentos e desportos. 0 oposto se dá com os professores de Educação Artística, que realizam atividades manuais, em algumas permanecendo muito tempo em uma mesma posição, além de carregarem material para suas aulas, como lápis de cor, pincéis, argila entre outros.

Assim, vemos a escola como mais um local de atuação para o fisioterapeuta, onde podem ser aplicados recursos fisioterapêuticos e ergonômicos, graça ao conhecimento desse profissional no campo da biomecânica, postura, antropometria, anatomia, fisiologia, entre outros. 0 fisioterapeuta pode atuar na análise dos equipamentos e mobiliários utilizados no dia a dia dos professores, além de interferir no uso inadequado da mecânica corporal. Outra área em que também pode atuar é no diagnóstico precoce e terapêutica específica com a finalidade de combater o aparecimento ou a evolução de sintomas osteomusculares. $(19,20)$.

\section{Conclusão}

Baseado nos dados expostos, o presente estudo apresentou alta prevalência de sintomas osteomusculares em professores da rede pública e privada de Pelotas, RS, destacando que a coluna vertebral foi o segmento corporal mais afetado. Além disso, o ambiente do trabalho proporcionado aos professores não apresenta uma ergonomia satisfatória. 
Sugere-se a realização de novos estudos para investigar outras variáveis, como a prática de atividade física, a implementação de fisioterapeuta nas escolas, ter filhos, o estado civil, entre outras, que podem influenciar a alta prevalência de sintomas osteomusculares nesta população. Outro ponto também a ser destacado é a necessidade de políticas escolares voltadas ao cuidado da saúde dos professores, bem como a inclusão do profissional fisioterapeuta em escolas, objetivando promover o bem-estar físico dos professores e alunos.

\section{Referências}

1. Oliveira CBE, Alves PB. Ensino fundamental: papel do professor, motivação e estimulação no contexto escolar. Paidéia. 2005;15(31):227-38.

2. Faria JIL, Casagrande LDR. A educação para o século XXI e a formação do professor reflexivo na enfermagem. Rev Latino-Am. Enfermagem. 2004;12(5):821-7.

3. Lemos JC. Cargas psíquicas no trabalho e processos de saúde em professores universitários [tese] Florianópolis: Universidade Federal de Santa Catarina. Centro Tecnológico; 2005.

4. Ministério da Educação. Instituto Nacional de Estudos e Pesquisas Educacionais Anísio Teixeira. Sinopse Estatística 2003. Brasília: INEP; 2003.

5. Sato L, Araújo MD, Udihara ML, Franco MA, Nicotera FN, Daldon MT, et al. Atividade em grupo com portadores de L. E. R. e achados sobre a dimensão psicossocial. Rev. Brasileira de Saúde Ocupacional. 1993: 79:49-62.

6. Nascimento GM. Estudo do absenteísmo dos trabalhadores de enfermagem em uma unidade básica e distrital de saúde do município de Ribeirão Preto - SP [dissertação]. Ribeirão Preto: Universidade de São Paulo; 2003.

7. Fernandes MH, da Rocha VM, Roncalli da CostaOliveira AG. Fatores associados à prevalência de sintomas osteomusculares em professores. Rev Salud Pública. 2009:11(2):256-267.

8. Porto LP, Reis IC, de Andrade JM, Nascimento CR, Carvalho FR. Doenças ocupacionais em professores atendidos pelo Centro de Estudos da Saúde do Trabalhador (CESAT). Rev Baiana de Saúde Pública. 2004: 28(1):33-49.
9. Normas Regulamentadoras de Ergonomia - NR 17: de 23/11/1990. [acesso em 14 mar. 2011]. Disponível em: http://www.mte.gov.br/legislacao/normas_ regulamentadoras/nr_17.pdf.

10. Penteado RZ, Pereira IMTB. Qualidade de vida e saúde vocal de professores. Rev Saúde Pública. 2007; 41(2):236-43.

11. Carvalho A, Alexandre NMC. Sintomas osteomusculares em professores do ensino fundamental. Rev Bras de Fisioter. 2006;10(1):35-41.

12. Pinheiro FA, Tróccoli BT, Carvalho CV. Validação do Questionário Nórdico de Sintomas Osteomusculares como medida de morbidade. Rev Saúde Pública. 2002;36(3):307-312.

13. Couto HA. Ergonomia aplicada ao trabalho. São Paulo: Ergo; 2001.

14. Brandão AG, Horta BL, Tomasi E. Sintomas de distúrbios osteomusculares em bancários de Pelotas e região: prevalência e fatores associados. Rev Bras Epidemiolog. 2005;8(3):295-305.

15. Gurgueira GP, Alexandre NMC, Filho HRC. Prevalência de sintomas músculo-esqueléticos em trabalhadoras de enfermagem. Rev Latino-Am Enfermagem. 2003; 11(5):608-13.

16. Picoloto D, Silveira Ed. Prevalência de sintomas osteomusculares e fatores associados em trabalhadores de uma indústria metalúrgica de Canoas - RS. Rev Ciênc Saúde Colet. 2008:13(2):507-16.

17. Oliveira ER. Prevalência de doenças osteomusculares em cirurgiões dentistas da rede publica e privada de Porto Velho - Rondônia [dissertação]. Brasília: Universidade de Brasília; 2007.

18. Dul J, Weerdmeester B. Ergonomia prática. 2a ed. São Paulo: Edgard Blücher; 2004.

19. de Miranda TEC, Vera RP, Pereira ER. Equipamento de apoio para membros superior: uma nova proposta ergonômica. Rev Bras Odontol. 2002;59(5):338-340.

20. Bau LMS. Fisioterapia do trabalho: ergonomia - legislação - reabilitação. Curitiba: Clã do Silva; 2002.

21. Ferreira EPV. "Onde está a margarida". Um estudo de caso sobre a mulher gari em Anicuns-GO [dissertação]. Anicuns: Faculdade de Educação e Ciências Humanas de Anicuns; 2005. 
22. Rocha LE, Debert-Ribeiro M. Trabalho, saúde e gênero: estudo comparativo sobre analistas de sistemas. Rev Saúde Pública. 2001;35(6):539-47.

23. Carvalho AJFP. Qualidade de vida e sintomas osteomusculares relacionados ao trabalho em professores do ensino fundamental. Rev Fisioter Bras. 2006; 7:279-84.

24. Rebelato JR, Morelli JG. Fisioterapia geriátrica: a prática da assistência ao idoso. São Paulo: Manole; 2004.

25. Silva GLF, Rosso AJ. As condições do trabalho docente dos professores das escolas públicas de Ponta Grossa, PR. Anais do $8^{\circ}$ Congresso Nacional de Educação EDUCERE; 2008.

26. Delcor NS, Araújo TM, Reis EJFB, Porto LA, Carvalho FM, Silva MO, et al. Condições de trabalho e saúde dos professores da rede particular de ensino de Vitória da Conquista, Bahia, Brasil. Cad Saúde Pública. 2004;20(1):187-96.

27. Cardoso JP, Ribeiro IQB, de Araújo TM, Carvalho FM, dos Reis EJFB. Prevalência de dor musculoesquelética em professores. Rev Bras Epidemiol. 2009; 12(4):604-14.
28. Reis EJFB, Carvalho FM, de Araújo TM, Porto LA, Silvany Neto AM. Trabalho e distúrbios psíquicos em professores da rede municipal de Vitória da Conquista, Bahia, Brasil. Cad Saúde Pública. 2005;21(5): 1480-1490.

29. Vasconcellos MD. O trabalho dos professores em questão. Educação Sociologia. 2002;23(81):307-11.

30. Gomes, L. Trabalho multifacetado de professores [dissertação]. Rio de Janeiro: Escola Nacional de Saúde, Fundação Osvaldo Cruz; 2002.

Recebido: $27 / 11 / 2010$

Received: $11 / 27 / 2010$

Aprovado: 10/02/2011

Approved: 02/10/2011 


\section{Anexos}

Tabela 1 - Região anatômica em relação a prevalência de sintomas osteomusculares e a incapacidade de exercer suas atividades normais nos últimos doze meses

\begin{tabular}{lccccccc}
\hline & \multicolumn{2}{c}{ Presença de sintoma osteomuscular } & & \multicolumn{2}{c}{ Incapacidade funcional } \\
\cline { 2 - 3 } \cline { 6 - 7 } Localização do sintoma & $\mathbf{N}$ & $\mathbf{\%}$ & $\mathbf{p}$-valor & & $\mathbf{N}$ & \% \\
\hline Pescoço & 163 & 50,9 & 0,00 & & 29 & 17,8 \\
Ombro & 167 & 45,7 & 0,00 & & 28 & 19 \\
Cotovelo & 38 & 11,9 & 0,52 & & 12 & 31,6 \\
Antebraço & 66 & 20,7 & 0,04 & & 25 & 37,9 \\
Punho/ mãos/ dedos & 123 & 38,5 & 0,00 & & 37 & 30,0 \\
Dorsal & 173 & 54,1 &, 000 & & 28 & 16,2 \\
Lombar & 157 & 49,1 &, 000 & & 30 & 19,1 \\
Quadril e/ou coxas & 41 & 12,8 &, 040 & & 9 & 2,8 \\
Joelhos & 90 & 28,1 &, 001 & & 21 & 6,5 \\
Tornozelo e pés & 76 & 23,8 &, 002 & & 20 & 26,4 \\
\hline
\end{tabular}

Tabela 2 - Prevalência de sintomas osteomusculares em relação às condições ergonômicas do trabalho apresentadas nas escolas públicas e privadas $(p=0,003)$

\begin{tabular}{|c|c|c|c|c|c|c|}
\hline \multirow[b]{2}{*}{ Ergonomia da sala de aula } & \multicolumn{2}{|c|}{ Presença de sintomas } & \multicolumn{2}{|c|}{ Escolas públicas } & \multicolumn{2}{|c|}{ Escolas privadas } \\
\hline & $\mathrm{N}$ & $\%$ & N & $\%$ & $\mathrm{~N}$ & $\%$ \\
\hline Excelente a boa & 56 & $78,9 \%$ & 53 & $22,1 \%$ & 18 & $22,5 \%$ \\
\hline Razoável & 100 & $88,5 \%$ & 77 & $32,1 \%$ & 36 & $45,0 \%$ \\
\hline Ruim a péssima & 131 & $96,3 \%$ & 110 & $45,8 \%$ & 26 & $32,5 \%$ \\
\hline Total & & & 240 & & 80 & \\
\hline
\end{tabular}

Tabela 3 - Prevalência de sintomas osteomusculares em relação à disciplina ministrada pelos professores

\begin{tabular}{|c|c|c|c|c|}
\hline \multirow[b]{2}{*}{ Disciplina } & \multicolumn{2}{|c|}{ Presença de sintomas } & \multicolumn{2}{|c|}{ Não apresentou sintomas } \\
\hline & $\mathrm{N}$ & $\%$ & N & $\%$ \\
\hline Português & 29 & 96,7 & 1 & 3,3 \\
\hline Matemática & 24 & 92,3 & 2 & 7,7 \\
\hline Geografia & 13 & 92,9 & 1 & 7,1 \\
\hline História & 19 & 95,0 & 1 & 5,0 \\
\hline Biologia/ Ciências & 19 & 86,4 & 3 & 13,6 \\
\hline Línguas Estrangeiras & 17 & 89,5 & 2 & 10,5 \\
\hline Ed. Física & 26 & 81,3 & 6 & 18,8 \\
\hline Ed. Artística & 17 & 100 & 0 & 0 \\
\hline Currículo ( $1^{\mathrm{a}}$ a $\left.4^{\mathrm{a}}\right)$ & 105 & 86,1 & 17 & 13,9 \\
\hline
\end{tabular}

